\title{
THE SMITH GROUP OF THE HYPERCUBE GRAPH
}

\author{
DAVID B. CHANDLER, PETER SIN* AND QING XIANG
}

\begin{abstract}
The $n$-cube graph is the graph on the vertex set of $n$ tuples of $0 \mathrm{~s}$ and $1 \mathrm{~s}$, with two vertices joined by an edge if and only if the $n$-tuples differ in exactly one component. We compute the Smith group of this graph, or, equivalently, the elementary divisors of an adjacency matrix of the graph.
\end{abstract}

*This work was partially supported by a grant from the Simons Foundation (\#204181 to Peter Sin). 


\section{INTRODUCTION}

Let $Q_{n}$ be the $n$-cube graph, with vertex set $\{0,1\}^{n}$ and two vertices joined if they differ in one component. In the language of association schemes, $Q_{n}$ is the distance 1 graph of the binary Hamming scheme.

It is of interest to compute linear algebraic invariants of a graph, such as its eigenvalues and the invariant factors of an adjacency matrix or Laplacian matrix. In the case of $Q_{n}$, previous work includes [1] and [2], where many of these invariants have been computed and some conjectures made about others. Here we shall consider the Smith group. If $X$ is an $m \times n$ integral matrix, then the Smith group of $X$ is the abelian group defined as the quotient of $\mathbb{Z}^{m}$ by the subgroup spanned by the columns $X$; that is, the abelian group whose invariant factor decomposition is given by the Smith normal form of $X$. If $A$ is the adjacency matrix (with respect to any ordering of the vertices) of a graph, then the Smith group of the graph is defined as the Smith group of $A$, and does not depend on the ordering on the vertices.

We recall that two integral matrices $X$ and $Y$ are integrally equivalent if there exist unimodular integral matrices $U$ and $V$ such that

$$
U X V=Y
$$

As is well known, $X$ and $Y$ are integrally equivalent if and only if $Y$ can be obtained from $X$ by a finite sequence of integral unimodular row and column operations. A diagonal form for $X$ is a matrix integrally equivalent to $X$ that has nonzero entries only on the leading diagonal. The Smith normal form of $X$ is one such diagonal form. Another way to describe the Smith group is in terms of the $p$-elementary divisors of $X$ with respect to primes $p$. Any diagonal form for $X$ gives a cyclic decomposition of the Smith group, so in a certain sense, the various diagonal forms carry the same information as the list of $p$-elementary divisors as $p$ varies over all primes.

The notion of integral equivalence can be generalized to $\mathbb{Z}_{(p)}$-equivalence, where $\mathbb{Z}_{(p)}$ is the ring of $p$-local integers, by requiring that the matrices $U$ and $V$ appearing in (1) be invertible over $\mathbb{Z}_{(p)}$. We can also consider the $p$-elementary divisors of any matrix $X$ with entries in $\mathbb{Z}_{(p)}$. If $X$ happens to have integer entries then its $p$-elementary divisors are the same whether it is considered as a matrix over $\mathbb{Z}$ or $\mathbb{Z}_{(p)}$

Let $A$ be an adjacency matrix for $Q_{n}$. It was proved in [2] that for every odd prime $p, A$ is $\mathbb{Z}_{(p)}$-equivalent to the diagonal matrix of the eigenvalues (all of which are integers). 
When $n$ is odd, all the eigenvalues are odd integers, so $\operatorname{det}(A)$ is an odd integer. Thus, the eigenvalue matrix is a diagonal form when $n$ is odd.

When $n$ is even, there remains the problem of finding the 2-elementary divisors of $A$. A conjecture for the multiplicity of each power $2^{e}$ as a 2-elementary divisor was stated in [2]. (See Conjecture 4.1 below.) The purpose of this paper is to give a proof of this conjecture. As a consequence of the conjecture, we obtain the following description of the Smith group of $Q_{n}$ when $n$ is even.

Theorem 1.1. Suppose that $n=2 m$ is even. Then the adjacency matrix $A$ of the $n$-cube $Q_{n}$ is integrally equivalent to a diagonal matrix with $\left(\begin{array}{c}n \\ m\end{array}\right)$ diagonal entries equal to zero and whose nonzero diagonal entries are the integers $k=1,2, \ldots m$, in which the multiplicity of $k$ is $2\left(\begin{array}{c}n \\ m-k\end{array}\right)$.

\section{INCLUSION OF SUBSETS OF A FINITE SET}

Let $n$ be a positive integer and $X=\{1,2, \ldots, n\}$. For brevity, we shall use the term $k$-subsets for the subsets of $X$ of size $k$. For $k \leq n$, let $M_{k}$ denote the free $\mathbb{Z}$-module on the set of $k$-subsets and for $t, k \leq n$ let

$$
\eta_{t, k}: M_{t} \rightarrow M_{k}
$$

be the incidence map, induced by inclusion. Thus, if $t \leq k$ a $t$-subset is mapped to the sum of all $k$-subsets containing it, while if $t \geq k$ the image of a $t$-subset is the sum of all $k$-subsets which it contains.

For each $k \leq n$, if we fix ordering on the $k$-subsets, we can think of elements of $\overline{M_{k}}$ as row vectors. Let $W_{t, k}$ denote the $\left(\begin{array}{l}n \\ t\end{array}\right) \times\left(\begin{array}{l}n \\ k\end{array}\right)$ matrix of $\eta_{t, k}$ with respect to these ordered bases of $M_{t}$ and $M_{k}$.

\section{Canonical Bases for subset modules}

The notion of the rank of a subset was introduced by Frankl [4]. We shall only need the concept of a $t$-subset of rank $t$, for $t \leq \frac{n}{2}$. let $T=\left\{i_{1}, i_{2}, \ldots, i_{t}\right\} \subseteq X$, with the elements in increasing order. Then $T$ has rank $t$ if and only if $i_{j} \geq 2 j$ for all $j=1, \ldots, t$. A $t$-subset is of rank $t$ if an only if it is the set of entries in the second row of a standard Young tableau of shape $[n-t, t]$. This is one way to see that the number of $t$-subsets of rank $t$ is $\left(\begin{array}{c}n \\ t\end{array}\right)-\left(\begin{array}{c}n \\ t-1\end{array}\right)$.

Assume $0 \leq j \leq k \leq \frac{n}{2}$. Let $E_{j, k}$ denote the $\left[\left(\begin{array}{c}n \\ j\end{array}\right)-\left(\begin{array}{c}n \\ j-1\end{array}\right)\right] \times\left(\begin{array}{l}n \\ k\end{array}\right)$ submatrix of $W_{j, k}$ formed from the the rows labeled by $j$-subsets of rank $j$, and let $E_{k}$ be the $\left(\begin{array}{l}n \\ k\end{array}\right) \times\left(\begin{array}{l}n \\ k\end{array}\right)$ matrix formed by stacking the $E_{j, k}$, $0 \leq j \leq k$, with $j$ increasing as we move down the matrix $E_{k}$. 
Wilson [5] found a diagonal form for $W_{t, k}$. We shall state his result for $t \leq k \leq n / 2$. There exist unimodular matrices $U_{t, k}$ and $V_{t, k}$ such that

$$
U_{t, k} W_{t, k} V_{t, k}=D_{t, k},
$$

where the diagonal form $D_{t, k}$ has diagonal entries $\left(\begin{array}{c}k-j \\ t-j\end{array}\right)$, with multiplicity $\left(\begin{array}{c}n \\ j\end{array}\right)-\left(\begin{array}{c}n \\ j-1\end{array}\right)$, for $j=0, \ldots, t$. Bier [3] refined Wilson's results, showing that we can take $U_{t, k}=E_{t}$ for all $k$ and $V_{t, k}=E_{k}{ }^{-1}$ for all $t$. The additional uniformity will be important for us.

Theorem 3.1. [3] Assume $k \leq \frac{n}{2}$. Then the matrix $E_{k}$ is unimodular. Furthermore, for $t \leq k$, we have

$$
E_{t} W_{t, k} E_{k}^{-1}=D_{t, k},
$$

where $D_{t, k}$ is Wilson's diagonal form.

We shall refer to the basis of $M_{k}$ corresponding to the rows of $E_{k}$ as the canonical basis of $M_{k}$. It consists of all vectors of the form $\eta_{j, k}(J)$, where $0 \leq j \leq k$ and $J$ is a $j$-subset of rank $j$.

\section{THE $n$-CUBE}

Let $Q_{n}$ denote the $n$-cube graph. The vertex set of $Q_{n}$ is $\{0,1\}^{n}$ and $\left(a_{1}, \ldots, a_{n}\right)$ is adjacent to $\left(b_{1}, \ldots, b_{n}\right)$ if and only if there is exactly one index $j$ with $a_{j} \neq b_{j}$. There is clearly a bijection of the vertex set with the set of subsets of $X=\{1,2, \ldots, n\}$, under which a vertex corresponds to the subset of indices where the vertex has entry 1 . We use this bijection and our fixed ordering of $k$-subsets for $k \leq n$ to order the vertices of $Q_{n}$, taking the subsets in order of increasing size. Let $A$ denote the adjacency matrix, with respect to this ordering.

Next we review the results of 2]. By viewing the vertex set of $Q_{n}$ as $\mathbb{F}_{2}^{n}$, and transforming $A$ by the character table of the additive group $\mathbb{F}_{2}^{n}$ the eigenvalues of $Q_{n}$ are found to be $n-2 \ell$, with multiplicity $\left(\begin{array}{l}n \\ \ell\end{array}\right)$ for $0 \leq \ell \leq n$.

When $n$ is odd, we see that $\operatorname{det} A$ is odd; hence all elementary divisors are odd. Then since $\mathbb{F}_{2}^{n}$ is an abelian 2-group, the same discrete Fourier transform method yields the elementary divisors. When $n$ is even, one still obtains the $p$-elementary divisors for all odd $p$. The 2-elementary divisors were not computed in [2], but the following conjecture was stated:

Conjecture 4.1. [2, 4.4.1] Suppose $n$ is even. Then the multiplicity of $2^{i}$ as a 2-elementary divisor of $A$ is equal to the number of eigenvalues of $A$ whose exact 2-power divisor is $2^{i+1}$. 


\section{BASES FOR THE FREE MODULE ON $Q_{n}$ AND MATRIX REPRESENTATIONS OF ADJACENCY}

Let $\mathbb{Z}^{Q_{n}}$ denote the free $\mathbb{Z}$-module on the set of vertices of $Q_{n}$. The matrix $A$ can be viewed as an endomorphism of $\mathbb{Z}^{Q_{n}}$, sending a vertex to the sum of all adjacent vertices. It is important for us to adopt a slightly different point of view. We can think of $\mathbb{Z}^{Q_{n}}$ as the ring of $\mathbb{Z}$-valued functions on the set of vertices of $Q_{n}$. Then the matrix $A$ defines the map $\alpha$ such that for any function $f \in \mathbb{Z}^{Q_{n}}$ we have

$$
\alpha(f)\left(a_{1}, \ldots, a_{n}\right)=\sum_{i=1}^{n} f\left(a_{1}, \ldots a_{i-1}, 1-a_{i}, a_{i+1}, \ldots, a_{n}\right),
$$

for $\left(a_{1}, \ldots, a_{n}\right) \in Q_{n}$. If we further regard the set $\{0,1\}^{n}$ of vertices of $Q_{n}$ as a subset of $\mathbb{Z}^{n}$, then functions are restrictions of polynomials and we have a ring isomorphism of $\mathbb{Z}^{Q_{n}}$ with

$$
\mathbb{Z}\left[X_{1}, \ldots, X_{n}\right] /\left(X_{i}^{2}-X_{i}, 1 \leq i \leq n\right) .
$$

Now a different natural basis becomes evident, namely the set of monomials $X_{I}=\prod_{i \in I} X_{i}$, for $I \subseteq X=\{1, \ldots, n\}$. With respect to the monomial basis we have

$$
\alpha\left(X_{I}\right)=\sum_{i \in I}\left(X_{I \backslash\{i\}}-X_{I}\right)+\sum_{i \notin I} X_{I}=(n-2|I|) X_{I}+\sum_{\substack{J \subset I \\|J|=|I|-1}} X_{J}
$$

Therefore, if we order monomials in the same way as we ordered subsets, the matrix of $\alpha$ with respect to this basis has the form

$\tilde{A}=\left[\begin{array}{c:c:c:c:c:c:c:c}n I & W_{0,1} & 0 & 0 & 0 & \ldots & 0 & 0 \\ \hdashline 0 & n-2) I & W_{1,2} & 0 & 0 & \ldots & 0 & 0 \\ \hdashline 0 & 0 & (n-4) I & W_{2,3} & 0 & \ldots & 0 & 0 \\ \hdashline 0 & 0 & 0 & (n-6) I & W_{3,4} & \ldots & 0 & 0 \\ \hdashline \vdots & \vdots & \ddots & \ddots & \ddots & \ddots & \vdots & \vdots \\ \hdashline 0 & 0 & \cdots & 0 & 0 & -(n-4) I & W_{n-2, n-1} & 0 \\ \hdashline 0 & 0 & \ldots & 0 & 0 & 0 & -(n-2) I & W_{n-1, n} \\ \hdashline 0 & 0 & \cdots & 0 & 0 & 0 & 0 & -n I\end{array}\right]$.

Assume from now on that $n=2 m$ is even.

$$
\tilde{A}=\left[\begin{array}{cc}
M & 0 \\
0 & N
\end{array}\right]
$$




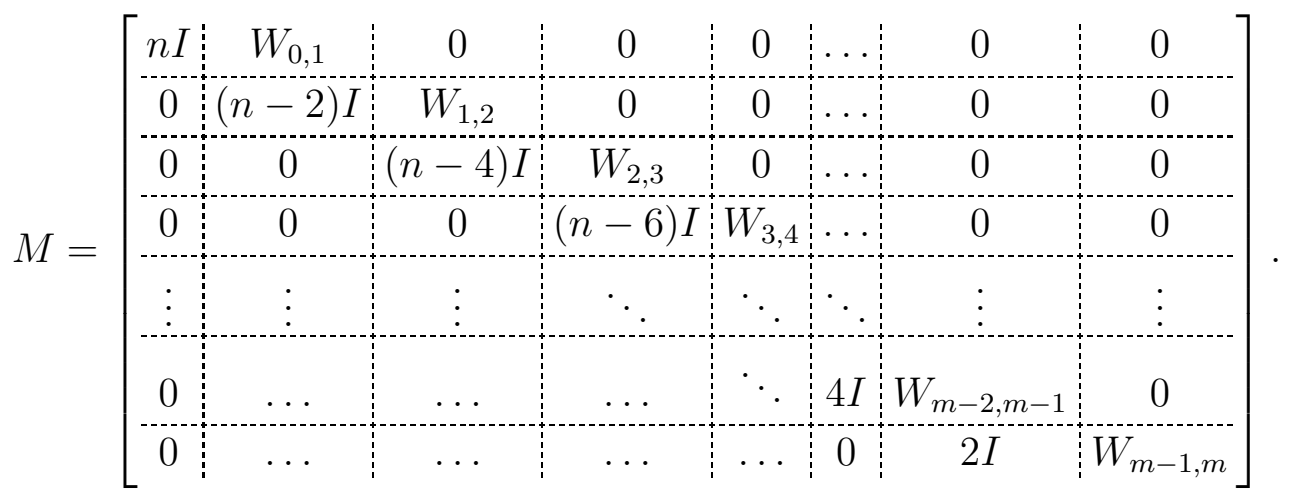

$N=\left[\begin{array}{c:c:c:c:c:c:c}W_{m, m+1} & 0 & \ldots & 0 & 0 & 0 & 0 \\ \hdashline-2 I & W_{m+1, m+2} & \ldots & 0 & 0 & 0 & 0 \\ \hdashline 0 & -4 I & \ddots & \vdots & \vdots & \vdots & \vdots \\ \hdashline \vdots & \ddots & \ddots & W_{n-4, n-3} & 0 & 0 & 0 \\ \hdashline \vdots & \vdots & \ddots & -(n-6) I & W_{n-3, n-2} & 0 & 0 \\ \hdashline \vdots & \vdots & \ldots & 0 & (-(n-4) I & W_{n-2, n-1} & 0 \\ \hdashline \vdots & \vdots & \ldots & 0 & 0 & -(n-2) I & W_{n-1, n} \\ \hdashline 0 & 0 & \ldots & 0 & 0 & 0 & -n I\end{array}\right]$.

Due to the block form ([6) of $\tilde{A}$, the multiplicity of a prime power as an elementary divisor of $\tilde{A}$ is the sum of its multiplicites in $M$ and $N$.

Up to now the choice of orderings on the $j$-susbsets, $0 \leq j \leq n$ used in the definition of the inclusion matrices $W_{t, k}$ has been an arbitrary (but fixed) one. Any choice would result in matrices of the form $M$ and $N$ as above, but the rows and columns of the submatrices $W_{t, k}$ would be permuted.

Now we shall specify these orderings more carefully. The matrix $M$ involves only the matrices $W_{t, k}$ with $0 \leq t<k \leq m$, while the matrix $N$ involves only the matrices $W_{t, k}$ with $m \leq t<k \leq n$. We start from the arbitrary but fixed ordering on the $j$-subsets with $0 \leq j \leq m$ that led to the matrix $M$. Then for $0 \leq j<m$ we choose the ordering of $(n-j)$-subsets to be the order induced by the complementation map. In this way we have specified an ordering on the $j$-subsets, for all $j$. Finally we wish to consider a second ordering on $m$-subsets, namely, the ordering defined from the given ordering by complementation. We use the first ordering on $m$-sets to define the submatrix $W_{m-1, m}$ of $M$ 
and the second ordering to define the submatrix $W_{m, m+1}$ of $N$. From the block form (6), we see that the matrix $\tilde{A}$ thus constructed differs from the one in which the same ordering on $m$-sets is used for both $W_{m-1, m}$ and $W_{m, m+1}$ only by a permutation of the rows in the first row-block of $N$, so the two matrices would be integrally equivalent.

The reason we have been careful to choose the ordering as above is that we now have, for $0 \leq t<k \leq m$,

$$
W_{n-k, n-t}=W_{k, t}^{t} \text {. }
$$

If we reverse the order of the block-rows and block-columns of $N$ and then take the transpose, we obtain

\begin{tabular}{|c|c|c|c|c|c|c|c|c|c|c|}
\hline \multirow{7}{*}{$N^{\prime}=$} & $-n I$ & $W_{n-1, n}^{t}$ & 0 & 0 & \multicolumn{2}{|c|}{0} & \multicolumn{3}{|c|}{0} & 0 \\
\hline & 0 & $-(n-2) I$ & $W_{n-2, n-1}^{t}$ & 0 & \multicolumn{2}{|c|}{0} & $\cdots$ & \multicolumn{2}{|l|}{0} & 0 \\
\hline & 0 & 0 & $-(n-4) I$ & $W_{n-3, n-2}^{t}$ & \multicolumn{2}{|c|}{0} & & \multicolumn{2}{|l|}{0} & 0 \\
\hline & 0 & 0 & 0 & $-(n-6) I$ & \multicolumn{2}{|c|}{$W_{n-4, n-3}^{t}$} & & \multicolumn{2}{|l|}{0} & 0 \\
\hline & $\vdots$ & $\vdots$ & . & $\cdot$ & \multicolumn{2}{|l|}{$\ddots$} & & & & $\vdots$ \\
\hline & 0 & $\ldots$ & $\ldots$ & $\ldots$ & & $-4 I$ & $W_{m+1}^{t}$ & $m+2$ & 0 \\
\hline & 0 & $\cdots$ & $\cdots$ & $\cdots$ & \multicolumn{2}{|c|}{. } & 0 & \multicolumn{2}{|c|}{$-2 I$} & $W_{m, r}^{t}$ \\
\hline \multirow{7}{*}{$=$} & $-n I$ & $W_{0,1}$ & 0 & 0 & \multicolumn{2}{|l|}{0} & \multicolumn{3}{|c|}{0} & 0 \\
\hline & 0 & $-(n-2) I$ & $W_{1,2}$ & 0 & 0 & & \multicolumn{3}{|c|}{0} & 0 \\
\hline & 0 & 0 & $-(n-4) I$ & $W_{2,3}$ & 0 & $\cdots$ & \multicolumn{3}{|c|}{0} & 0 \\
\hline & 0 & 0 & 0 & $-(n-6) I$ & $W_{3,4}$ & $\ldots$ & \multicolumn{3}{|c|}{0} & 0 \\
\hline & $\vdots$ & $\vdots$ & $\vdots$ & $\cdot$ & & & & $\vdots$ \\
\hline & 0 & . & . & & & $-4 I$ & \multicolumn{2}{|c|}{$W_{m-2, m-1}$} & & 0 \\
\hline & 0 & $\cdots$ & $\cdots$ & $\cdots$ & $\cdots$ & 0 & \multicolumn{2}{|c|}{$-2 I$} & $W_{m}$ & $-1, m$ \\
\hline
\end{tabular}

Thus, $N^{\prime}$ differs from $M$ only by the sign of the diagonal entries. In fact, to see that $N^{\prime}$ is integrally equivalent to $M$, we perform the following simple sequence of unimodular operations. First mutliply the first block-column by -1 , then multiply the second block-row by -1 , then the third block-column, etc., until we reach the bottom-right of the matrix, at which point $N^{\prime}$ has been converted to $M$.

We have established the following reduction.

Lemma 5.1. Let $M$ and $N$ be the matrices in ([6). Then $M$ and $N^{t}$ are integrally equivalent. In particular the multiplicity of an elementary 
divisor of $\tilde{A}$ (and hence of $A$ ) is twice its multplicity as an elementary divisor of $M$.

From now, we focus on the matrix $M$. Let $E_{j}$ for $0 \leq j \leq m$ be defined as in $\S 3$ and for $0 \leq k \leq m$, set

$$
E(k)=\operatorname{diag}\left(E_{0}, E_{1}, \ldots, E_{k}\right) .
$$

Then from Theorem 3.1 we immediately obtain:

$$
E(m-1) \cdot M \cdot E(m)^{-1}=\left[\begin{array}{c:c:c:c:c:c:c:c}
n I & D_{0,1} & 0 & 0 & 0 & \ldots & 0 & 0 \\
\hdashline 0 & (n-2) I & D_{1,2} & 0 & 0 & \ldots & 0 & 0 \\
\hdashline 0 & 0 & (n-4) I & D_{2,3} & 0 & \ldots & 0 & 0 \\
\hdashline 0 & 0 & 0 & n-6) I & D_{3,4} & \ldots & 0 & 0 \\
\hdashline \vdots & \vdots & \vdots & \ddots & \ddots & \ddots & \vdots & \vdots \\
\hdashline 0 & \ldots & \ldots & \ldots & \ddots & 4 I & D_{m-2, m-1} & 0 \\
\hdashline 0 & \cdots & \cdots & \cdots & \cdots & 0 & 2 I & D_{m-1, m}
\end{array}\right] .
$$

For example when $n=4$, we have

$$
M=\left[\begin{array}{c:cccc:cccccc}
4 & 1 & 1 & 1 & 1 & 0 & 0 & 0 & 0 & 0 & 0 \\
\hdashline 0 & 2 & 0 & 0 & 0 & 1 & 1 & 0 & 1 & 0 & 0 \\
0 & 0 & 2 & 0 & 0 & 1 & 0 & 1 & 0 & 1 & 0 \\
0 & 0 & 0 & 2 & 0 & 0 & 1 & 1 & 0 & 0 & 1 \\
0 & 0 & 0 & 0 & 2 & 0 & 0 & 0 & 1 & 1 & 1
\end{array}\right]
$$

$$
E(1)=\left[\begin{array}{c:cccc}
1 & 0 & 0 & 0 & 0 \\
\hdashline 0 & 1 & 1 & 1 & 1 \\
0 & 0 & 1 & 0 & 0 \\
0 & 0 & 0 & 1 & 0 \\
0 & 0 & 0 & 0 & 1
\end{array}\right]
$$




$$
E(2)=\left[\begin{array}{c:cccc:cccccc}
1 & 0 & 0 & 0 & 0 & 0 & 0 & 0 & 0 & 0 & 0 \\
\hdashline 0 & 1 & 1 & 1 & 1 & 0 & 0 & 0 & 0 & 0 & 0 \\
0 & 0 & 1 & 0 & 0 & 0 & 0 & 0 & 0 & 0 & 0 \\
0 & 0 & 0 & 1 & 0 & 0 & 0 & 0 & 0 & 0 & 0 \\
0 & 0 & 0 & 0 & 1 & 0 & 0 & 0 & 0 & 0 & 0 \\
\hdashline 0 & 0 & 0 & 0 & 0 & 1 & 1 & 1 & 1 & 1 & 1 \\
0 & 0 & 0 & 0 & 0 & 1 & 0 & 1 & 0 & 1 & 0 \\
0 & 0 & 0 & 0 & 0 & 0 & 1 & 1 & 0 & 0 & 1 \\
0 & 0 & 0 & 0 & 0 & 0 & 0 & 0 & 1 & 1 & 1 \\
0 & 0 & 0 & 0 & 0 & 0 & 0 & 0 & 0 & 1 & 0 \\
0 & 0 & 0 & 0 & 0 & 0 & 0 & 0 & 0 & 0 & 1
\end{array}\right]
$$

We denote the matrix in (을 by $B$ and let $B^{\prime}$ be the matrix obtained by zeroing out the diagonal. Thus,

$$
B^{\prime}=\left[\begin{array}{c:c:c:c:c:c:c}
0 & D_{0,1} & 0 & 0 & 0 & 0 & \ldots \\
\hdashline 0 & 0 & D_{1,2} & 0 & 0 & 0 & \ldots \\
\hdashline 0 & 0 & 0 & D_{2,3} & 0 & 0 & \ldots \\
\hdashline 0 & 0 & 0 & 0 & D_{3,4} & 0 & \ldots \\
\hdashline \vdots & \vdots & \vdots & \vdots & \ddots & \ddots & \ddots \\
0 & \cdots & 0 & 0 & 0 & 0 & D_{m-1, m}
\end{array}\right] .
$$

We examine the matrix $D_{i-1, i}$ more closely. It has $\left(\begin{array}{c}n \\ i-1\end{array}\right)$ rows and $\left(\begin{array}{l}n \\ i\end{array}\right)$ columns and has the form

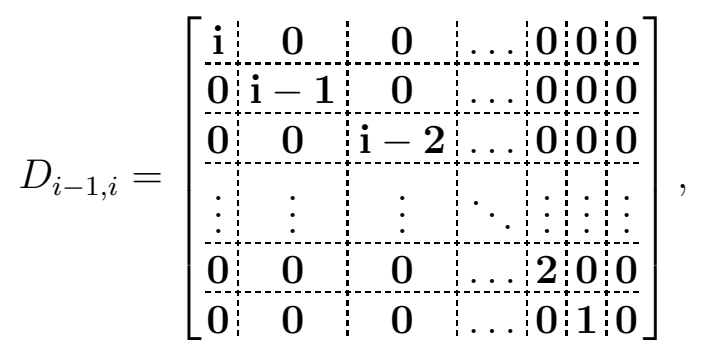

where a bold number $\mathbf{s}, s \neq 0$ represents a scalar matrix $s I$ of the appropriate size and $\mathbf{0}$ denotes a zero block of the appropriate size. 
The block sizes are readily found; if $n_{j}=\left(\begin{array}{c}n \\ j\end{array}\right)-\left(\begin{array}{c}n \\ j-1\end{array}\right)$, then $D_{i-1, i}$ has $i+1$ block-columns and $i$ block-rows, and the $k$-th block-column of $D_{i-1, i}$ contains $n_{k-1}$ columns, for $1 \leq k \leq i+1$, while for $1 \leq \ell \leq i$, the $\ell$-th block-row contains $n_{\ell-1}$ rows.

Also, let (c) denote a scalar matrix (more precisely the class of scalar matrices) whose scalar is a multiple of $c$. (We introduce this notation because all that we shall use about the diagonal entries is that they are even, and working with the entire class of such matrices will facilitate the use of mathematical induction.) Then we can write $B$ in the following form (more precisely, $B$ lies in the given class of matrices). 


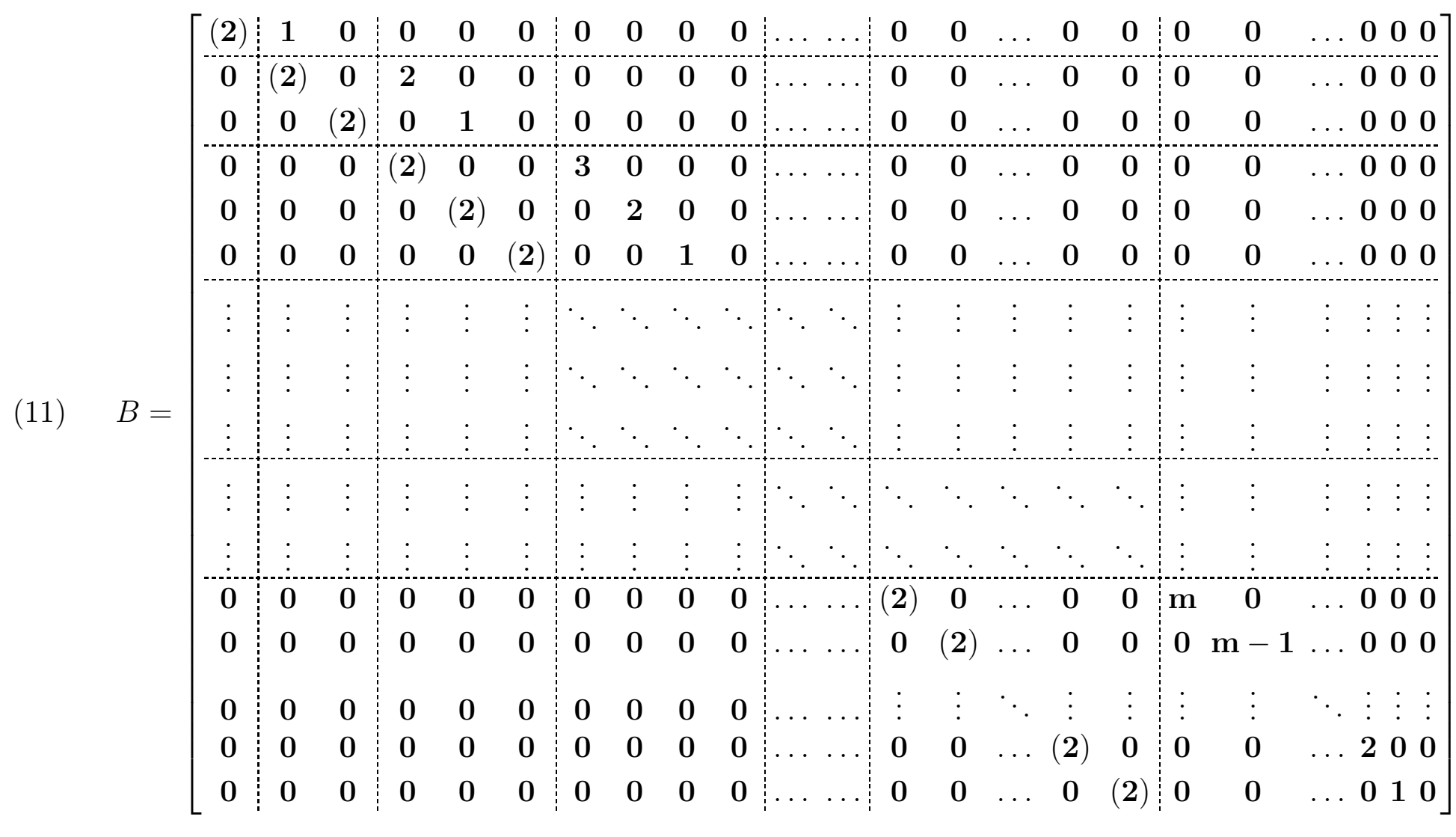


Lemma 5.2. $B$ and $B^{\prime}$ are equivalent over the 2-local integers $\mathbb{Z}_{(2)}$.

Proof. Let $\bar{B}(m)$ denote a matrix (actually the class of matrices) in which all the blocks (bold numbers) in $B$ are replaced by the plain numbers, and each $(\mathbf{c})$ is replaced by $(c)$, representing a multiple of the 2-local integer $c$. It suffices to show that any matrix of the form $B(m)$ is 2-locally equivalent to the matrix obtained by zeroing out its diagonal. Indeed, if there is a sequence of $\mathbb{Z}_{(2)}$-unimodular row and column operations which zero out the diagonal of $\bar{B}(m)$, then the same sequence of the blockwise versions of these operations will kill the diagonal of $B$. So we may discard $B$ and work only with $\bar{B}(m)$ from now on.

$$
\bar{B}(m)=\left[\begin{array}{c:c:c:c:c:c:c:c}
(2) I & \bar{D}_{1} & 0 & 0 & 0 & \ldots & 0 & 0 \\
\hdashline 0 & (2) I & \bar{D}_{2} & 0 & 0 & \ldots & 0 & 0 \\
\hdashline 0 & 0 & (2) I & \bar{D}_{3} & 0 & \ldots & 0 & 0 \\
\hdashline 0 & 0 & 0 & (2) I & \bar{D}_{4} & \ldots & 0 & 0 \\
\hdashline \vdots & \vdots & \vdots & \ddots & \ddots & \ddots & \vdots & \vdots \\
\hdashline 0 & \ldots & \ldots & \ldots & \ddots & (2) I & \bar{D}_{m-1} & 0 \\
\hdashline 0 & \ldots & \ldots & \ldots & \ldots & 0 & (2) I & \bar{D}_{m}
\end{array}\right],
$$

where

$$
\bar{D}_{i}=\left[\begin{array}{cccccc:c}
i & 0 & 0 & \ldots & 0 & 0 & 0 \\
0 & i-1 & 0 & \ldots & 0 & 0 & 0 \\
0 & 0 & i-2 & \ldots & 0 & 0 & 0 \\
\vdots & \vdots & \vdots & \ddots & \vdots & \vdots & \vdots \\
0 & 0 & 0 & \ldots & 2 & 0 & 0 \\
0 & 0 & 0 & \ldots & 0 & 1 & 0
\end{array}\right]
$$

is the "condensed" version of $D_{i-1, i}$, consisting of a diagonal $i \times i$ matrix augmented by a column of zeros.

From now on it will be convenient to refer to the entries of $\bar{B}(m)$ (and matrices derived from it) by their positions relative to the submatrices $\bar{D}_{i}$. Note this is a change in the way we are indexing blocks, compared to how we did it in the original matrix $M$. The row of the main matrix containing the $k$-th row of $\bar{D}_{i}$ is assigned the label $[i, k]$ and the column containing the $\ell$-th column of $\bar{D}_{j}$ is assigned the label $[j, \ell]$, while the first column is labeled $[0,1]$. Thus, in a row index $[i, k]$ we 
have $1 \leq i \leq m$ and $1 \leq k \leq i$, while a column index $[j, \ell]$ has $0 \leq j \leq m$ and $1 \leq \ell \leq j+1$.

We shall perform some row and column operations using the odd entries on the main diagonals of the $\bar{D}_{i}$ s to kill diagonal entries of the main matrix. Each odd entry of $\bar{D}_{i}$ for $1 \leq i \leq m-1$ will be used to kill the two diagonal entries of the main matrix, one in the same row as the odd entry and one in the same column. The odd entries of $\bar{D}_{m}$ will be used to kill the diagonal entry in the same row. This procedure will create new entries (4) at locations where there previously were zeroes. To be precise, if the odd entry is at $([i, k],[i, k])$ then the diagonal entry of the main matrix in the same row is at $([i, k],[i-1, k])$. The diagonal entry of the main matrix in the same column as $([i, k],[i, k])$ is at $([i+1, k],[i, k]$ ) (with no such entry if $i=m$ ). By multiplying column $[i, k]$ by a suitable element of (2) and subtracting it from column $[i-1, k]$, we kill off the entry at $([i, k],[i-1, k])$ and create a new entry (4) at $([i+1, k],[i-1, k)$, if $i \leq m-1$. The new entry is (4) because the entry being subtracted from zero is a (2)-multiple of the (2) at $([i+1, k],[i, k])$ on the main diagonal. No new entry is created if $i=m$. Then, we can subtract a (2)-multiple of row $[i, k]$ from row $[i+1, k]$ to kill off the diagonal entry at $([i+1, k],[i, k])$, without creating any new nonzero entries, if $i \leq m-1$, while there is nothing to be done when $i=m$. The following figure (for $m=5$ ) shows the resulting matrix after performing these operations with the entry 1 at $([3,3],[3,3])$. The block indices are specified by numbered braces. 


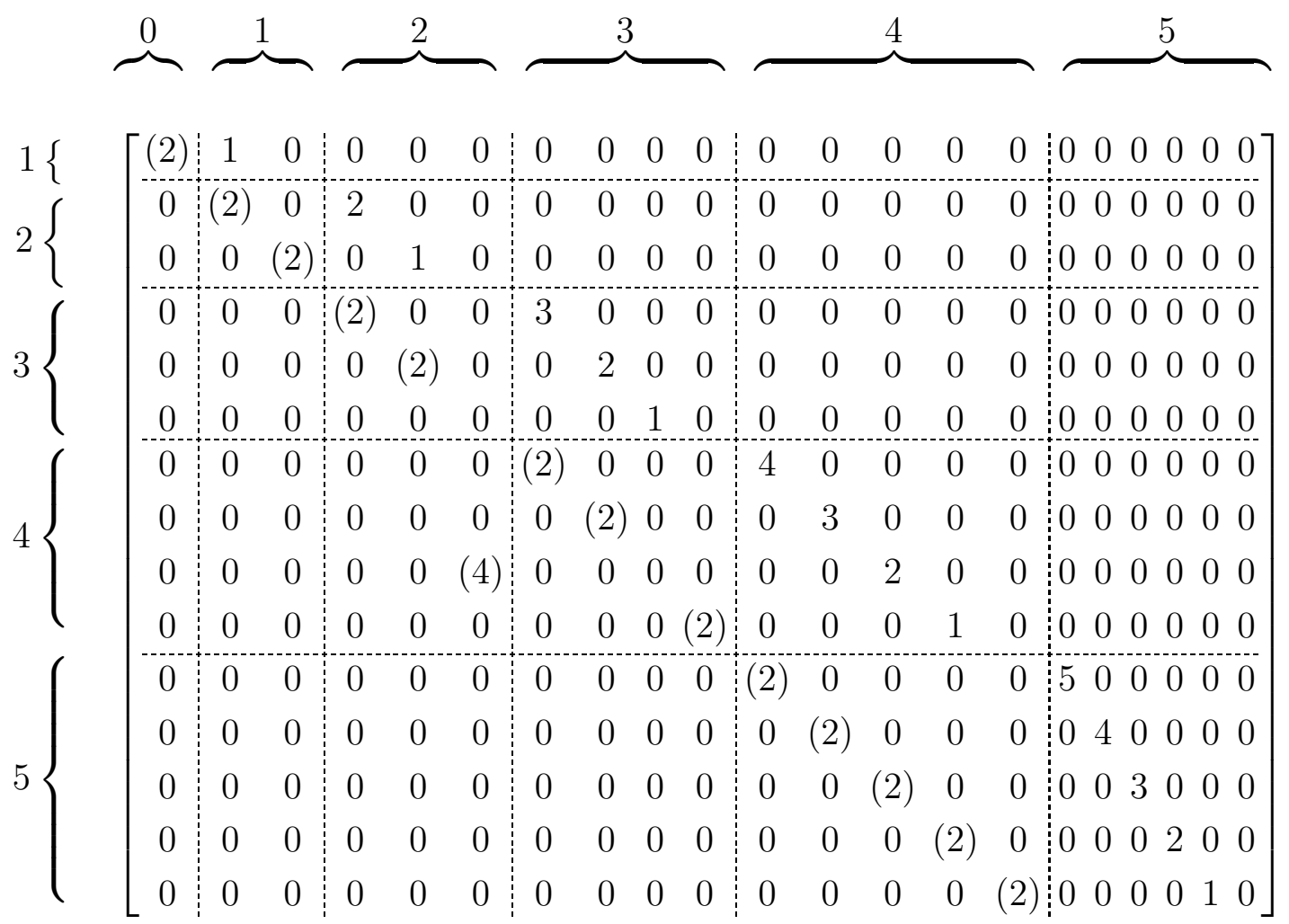

We do this for all odd entries of all the $\bar{D}_{i}$. In our $m=5$ example, after the operations the matrix looks as follows.

$\left[\begin{array}{c:cc:ccc:cccc:cccc:cccccccccc}0 & 1 & 0 & 0 & 0 & 0 & 0 & 0 & 0 & 0 & 0 & 0 & 0 & 0 & 0 & 0 & 0 & 0 & 0 & 0 & 0 \\ \hdashline(4) & 0 & 0 & 2 & 0 & 0 & 0 & 0 & 0 & 0 & 0 & 0 & 0 & 0 & 0 & 0 & 0 & 0 & 0 & 0 & 0 \\ 0 & 0 & 0 & 0 & 1 & 0 & 0 & 0 & 0 & 0 & 0 & 0 & 0 & 0 & 0 & 0 & 0 & 0 & 0 & 0 & 0 \\ \hdashline 0 & 0 & 0 & 0 & 0 & 0 & 3 & 0 & 0 & 0 & 0 & 0 & 0 & 0 & 0 & 0 & 0 & 0 & 0 & 0 & 0 \\ 0 & 0 & (4) & 0 & 0 & 0 & 0 & 2 & 0 & 0 & 0 & 0 & 0 & 0 & 0 & 0 & 0 & 0 & 0 & 0 & 0 \\ 0 & 0 & 0 & 0 & 0 & 0 & 0 & 0 & 1 & 0 & 0 & 0 & 0 & 0 & 0 & 0 & 0 & 0 & 0 & 0 & 0 \\ \hdashline 0 & 0 & 0 & (4) & 0 & 0 & 0 & 0 & 0 & 0 & 4 & 0 & 0 & 0 & 0 & 0 & 0 & 0 & 0 & 0 & 0 \\ 0 & 0 & 0 & 0 & 0 & 0 & 0 & 0 & 0 & 0 & 0 & 3 & 0 & 0 & 0 & 0 & 0 & 0 & 0 & 0 & 0 \\ 0 & 0 & 0 & 0 & 0 & (4) & 0 & 0 & 0 & 0 & 0 & 0 & 2 & 0 & 0 & 0 & 0 & 0 & 0 & 0 & 0 \\ 0 & 0 & 0 & 0 & 0 & 0 & 0 & 0 & 0 & 0 & 0 & 0 & 0 & 1 & 0 & 0 & 0 & 0 & 0 & 0 & 0 \\ \hdashline 0 & 0 & 0 & 0 & 0 & 0 & 0 & 0 & 0 & 0 & 0 & 0 & 0 & 0 & 0 & 5 & 0 & 0 & 0 & 0 & 0 \\ 0 & 0 & 0 & 0 & 0 & 0 & 0 & (4) & 0 & 0 & 0 & 0 & 0 & 0 & 0 & 0 & 4 & 0 & 0 & 0 & 0 \\ 0 & 0 & 0 & 0 & 0 & 0 & 0 & 0 & 0 & 0 & 0 & 0 & 0 & 0 & 0 & 0 & 0 & 3 & 0 & 0 & 0 \\ 0 & 0 & 0 & 0 & 0 & 0 & 0 & 0 & 0 & (4) & 0 & 0 & 0 & 0 & 0 & 0 & 0 & 0 & 2 & 0 & 0 \\ 0 & 0 & 0 & 0 & 0 & 0 & 0 & 0 & 0 & 0 & 0 & 0 & 0 & 0 & 0 & 0 & 0 & 0 & 0 & 1 & 0\end{array}\right]$


At this point, the rows and columns of the main matrix that correspond to an odd entry of some $\bar{D}_{i}$ have no other nonzero entries. The submatrix $D(m)$ formed from these rows and columns is a square diagonal matrix with odd entries, and the main matrix is the block sum of this diagonal matrix with the submatrix $A(m)$ formed from the remaining rows and columns. (Since permuting rows and columns results in an integrally equivalent matrix, the reader may find it helpful, for easier visualization of this block sum decomposition, to renumber the rows and columns in order to put $D(m)$ at the bottom right of the main matrix.) We observe that $A(m)$ will have entries (4) all along its main diagonal, and each row of $A(m)$ has one nonzero entry off the main diagonal (coming from the even entries on the main diagonals of the $\bar{D}_{i}$.) In the picture below, we show $A(5)$.

$$
\left[\begin{array}{c:c:cc:cc:ccc:ccc}
(4) & 0 & 2 & 0 & 0 & 0 & 0 & 0 & 0 & 0 & 0 & 0 \\
\hdashline 0 & (4) & 0 & 0 & 2 & 0 & 0 & 0 & 0 & 0 & 0 & 0 \\
\hdashline 0 & 0 & (4) & 0 & 0 & 0 & 4 & 0 & 0 & 0 & 0 & 0 \\
0 & 0 & 0 & (4) & 0 & 0 & 0 & 2 & 0 & 0 & 0 & 0 \\
\hdashline 0 & 0 & 0 & 0 & (4) & 0 & 0 & 0 & 0 & 4 & 0 & 0 \\
0 & 0 & 0 & 0 & 0 & (4) & 0 & 0 & 0 & 0 & 2 & 0
\end{array}\right]
$$

We next note that if $i$ and $j$ have different parity then all entries at locations $([i, k],[j, \ell])$ are zero, so $A(m)$ is the block sum of the submatrix $A^{\prime \prime}(m)$ corresponding to rows $[i, k]$ and columns $[j, \ell]$ for odd indices $i$ and $j$, and the submatrix $A^{\prime}(m)$ formed from the rows and columns corresponding to the even indices. For better visualization, we can reorder the rows and columns. The block decomposition of $A(5)$ after reording rows and columns is shown below.

$$
\left[\begin{array}{c:cc:ccc:c:cc:ccc}
(4) & 2 & 0 & 0 & 0 & 0 & 0 & 0 & 0 & 0 & 0 & 0 \\
\hdashline 0 & (4) & 0 & 4 & 0 & 0 & 0 & 0 & 0 & 0 & 0 & 0 \\
0 & 0 & (4) & 0 & 2 & 0 & 0 & 0 & 0 & 0 & 0 & 0 \\
\hdashline 0 & 0 & 0 & 0 & 0 & 0 & (4) & 2 & 0 & 0 & 0 & 0 \\
\hdashline 0 & 0 & 0 & 0 & 0 & 0 & 0 & (4) & 0 & 4 & 0 & 0 \\
0 & 0 & 0 & 0 & 0 & 0 & 0 & 0 & (4) & 0 & 2 & 0
\end{array}\right]
$$

We can see from this picture that $A^{\prime}(5)=2 \bar{B}(2)=A^{\prime \prime}(5)$. In general, it is easy to see that if we delete the rows and columns of $\bar{D}_{i}$ which contain odd entries, the resulting matrix is $2 \bar{D}_{i^{\prime}}$ where $i^{\prime}=\left\lfloor\frac{i}{2}\right\rfloor$ (with the understanding that $\bar{D}_{0}$ is the empty matrix). It follows 
that $A^{\prime}(m)=2 \bar{B}\left(m^{\prime}\right)$, where $m^{\prime}=\left\lfloor\frac{m}{2}\right\rfloor$, and $A^{\prime \prime}(m)=2 \bar{B}\left(m^{\prime \prime}\right)$, and $m^{\prime \prime}=\left\lfloor\frac{m-1}{2}\right\rfloor$. (More formally, $A^{\prime}(m)$ belongs to the class $2 B\left(m^{\prime}\right)$ and $A^{\prime \prime}(m)$ belongs to the class $2 B\left(m^{\prime \prime}\right)$.)

Thus, we have shown that $\bar{B}(m)$ is 2-locally equivalent to a matrix $C$ which is the diagonal block sum of $2 \bar{B}\left(m^{\prime}\right)$ and $2 \bar{B}\left(m^{\prime \prime}\right)$ and the diagonal matrix $D(m)$. Arguing by induction on $m$, there are $\mathbb{Z}_{(2)^{-}}$ unimodular row and column operations on $C$ that kill the diagonals of $A^{\prime}(m)$ and of $A^{\prime \prime}(m)$ while leaving all other entries of $C$ unchanged. The resulting matrix is equal, up to reordering rows and columns, to the matrix obtained from $\bar{B}(m)$ by zeroing out its diagonal.

Theorem 5.3. Suppose that $n=2 m$ is even. Then the adjacency matrix $A$ of the $n$-cube $Q_{n}$ is $\mathbb{Z}_{(2)}$-equivalent to a diagonal form with $\left(\begin{array}{l}n \\ m\end{array}\right)$ diagonal entries equal to zero and whose nonzero diagonal entries are the integers $k=1,2, \ldots m$, in which the multiplicity of $k$ is $2\left(\begin{array}{c}n \\ m-k\end{array}\right)$.

Proof. By Lemma 5.1, it suffices to find a diagonal form for $M$, hence for $B^{\prime}$, with which we have shown $M$ to be integrally equivalent. The nonzero entries of $B^{\prime}$ are the integers 1 to $m$. The integer $k$ occurs in $D_{i-1, i}$ only when $k \leq i$ and then its multiplicity in $D_{i-1, i}$ is $\left(\begin{array}{c}n \\ i-k\end{array}\right)-$ $\left(\begin{array}{c}n \\ i-1-k\end{array}\right)$. Therefore, the multiplicity of $k$ in $B^{\prime}$ is

$$
\sum_{i=k}^{m}\left(\begin{array}{c}
n \\
i-k
\end{array}\right)-\left(\begin{array}{c}
n \\
i-1-k
\end{array}\right)=\sum_{s=0}^{m-k}\left(\begin{array}{c}
n \\
s
\end{array}\right)-\left(\begin{array}{c}
n \\
s-1
\end{array}\right)=\left(\begin{array}{c}
n \\
m-k
\end{array}\right) .
$$

Corollary 5.4. Conjecture 4.1 is true.

By Lemma 5.1, the multiplicity of the prime power $p^{e}$ as an $p$ elementary divisor of $\tilde{A}$, and hence also of $A$, is twice the sum of the binomial coefficients $\left(\begin{array}{c}n \\ m-k\end{array}\right)$, taken over those $k$ with $1 \leq k \leq m$ that are exactly divisible by $p^{e}$. On the other hand, we know $(\S 4)$ that the nonzero eigenvalues of $A$ are the integers $\pm 2 k$ for $1 \leq k \leq m$, and the multiplicity of $2 k$ is $\left(\begin{array}{c}n \\ m-k\end{array}\right)$. Comparing these numbers for $p=2$, we see that Conjecture 4.1 is true.

5.1. Proof of Theorem 1.1. By Theorem 5.3, it suffices to show, for every odd prime $p$, that $A$ is $\mathbb{Z}_{(p)}$-equivalent to a diagonal matrix whose nonzero entries are $k=1, \ldots, m$, where $k$ has multiplicity $2\left(\begin{array}{c}n \\ m-k\end{array}\right)$. Let $p$ be given. We know from [2] that $A$ is $\mathbb{Z}_{(p)}$-equivalent to a diagonal matrix whose nonzero are $n-2 \ell$ with multiplicity $\left(\begin{array}{l}n \\ \ell\end{array}\right)$, for $0 \leq \ell \leq n$. The latter is easily seen to be integrally equivalent to a diagonal matrix whose nonzero entries are $2 k=1, \ldots, m$, where $k$ has multiplicity 
$2\left(\begin{array}{c}n \\ m-k\end{array}\right)$, and hence $\mathbb{Z}_{(p)}$-equivalent to the diagonal form given in Theorem 1.1.

5.2. Final remarks. It would be of interest to find a diagonal form for the Laplacian matrix $n I-A$ of $Q_{n}$. The Smith group of this matrix is called the critical group of $Q_{n}$. By the results of [1], only the 2-Sylow subgroup of the critical group remains to be determined, for both odd and even $n$. We do not have any conjecture about its exact structure. However, we note that if two integral matrices are equal modulo $p^{s}$ then for $i<s$ the multiplicity of $p^{i}$ as a $p$-elementary divisor is the same for both matrices. Thus, for example, when $n=2^{s}$, Theorem 5.3 gives part of the cyclic decomposition of the 2-Sylow subgroup of the critical group.

\section{REFERENCES}

[1] Hua Bai, On the critical group of the n-cube. Linear Algebra Appl. 369 (2003) 251-261.

[2] J. Ducey, D. Jalil, Integer invariants of abelian Cayley graphs, Linear Algebra Appl. 445 (2014) 316-325

[3] Thomas Bier, Remarks on recent formulas of Wilson and Frankl, European J. Combin. 14 (1993), no. 1, 1-8.

[4] P. Frankl, Intersection theorems and mod p rank of inclusion matrices, Journal of Combinatorial Theory, Series A, 54 (1990), 85-94

[5] Richard M. Wilson, A diagonal form for the incidence matrices of $t$-subsets vs. k-subsets, European J. Combin. 11 (1990), no. 6, 609-615.

6 Georgian Circle, Newark, DE 19711, USA

Department of Mathematics, University of Florida, P. O. Box 118105, Gainesville, FL 32611, USA

Department of Mathematical Sciences, University of Delaware, Newark, DE 19716, USA 